Braz J Med Biol Res, September 2011, Volume 44(9) 848-854

doi: 10.1590/S0100-879X2011007500102

Cardiovascular and autonomic modulation by the central nervous system after aerobic exercise training

M.C. Martins-Pinge

The Brazilian Journal of Medical and Biological Research is partially financed by

\section{足CNPq}

da Ciência Ministério

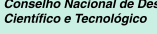

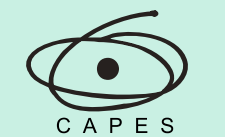

Ministério
da Educação

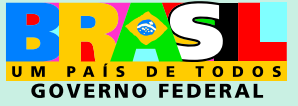

D JAPESP

\author{
Institutional Sponsors
}
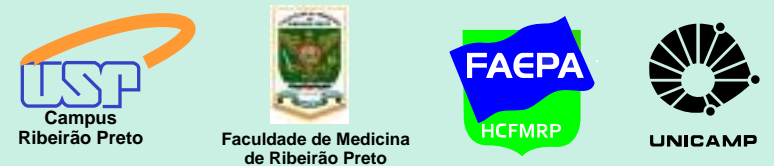

๑ SHIMADZU

Explore High - Performance MS In Protitrap Technology

analitica Thermo 


\title{
Cardiovascular and autonomic modulation by the central nervous system after aerobic exercise training
}

\author{
M.C. Martins-Pinge
}

Laboratório de Fisiologia Cardiovascular, Departamento de Ciências Fisiológicas, Centro de Ciências Biológicas, Universidade Estadual de Londrina, Londrina, PR, Brasil

\begin{abstract}
The autonomic nervous system plays a key role in maintaining homeostasis under normal and pathological conditions. The sympathetic tone, particularly for the cardiovascular system, is generated by sympathetic discharges originating in specific areas of the brainstem. Aerobic exercise training promotes several cardiovascular adjustments that are influenced by the central areas involved in the output of the autonomic nervous system. In this review, we emphasize the studies that investigate aerobic exercise training protocols to identify the cardiovascular adaptations that may be the result of central nervous system plasticity due to chronic exercise. The focus of our study is on some groups of neurons involved in sympathetic regulation. They include the nucleus tractus solitarii, caudal ventrolateral medulla and the rostral ventrolateral medulla that maintain and regulate the cardiac and vascular autonomic tonus. We also discuss studies that demonstrate the involvement of supramedullary areas in exercise training modulation, with emphasis on the paraventricular nucleus of the hypothalamus, an important area of integration for autonomic and neuroendocrine responses. The results of these studies suggest that the beneficial effects of physical activity may be due, at least in part, to reductions in sympathetic nervous system activity. Conversely, with the recent association of physical inactivity with chronic disease, these data may also suggest that increases in sympathetic nervous system activity contribute to the increased incidence of cardiovascular diseases associated with a sedentary lifestyle.
\end{abstract}

Key words: Brainstem; Hypothalamus; Aerobic exercise training; Rostroventrolateral medulla; Paraventricular nucleus; Nucleus tractus solitarii

\section{Introduction}

Maintaining blood pressure (BP) within a normal range depends on a fine regulation of cardiac output and peripheral resistance. Different control mechanisms are involved in maintaining the moment-to-moment variation in $\mathrm{BP}$, acting on the regulation of caliber and vascular reactivity and on cardiac output. The study of mechanisms controlling BP has identified many physiological systems that interact in a complex manner and with redundancy to ensure that BP is at adequate levels in the most diverse situations (1). One of the most important systems activated is the autonomic nervous system (ANS).

The ANS plays a key role in maintaining homeostasis under normal and pathological conditions (2). The sympathetic tone, particularly for the cardiovascular system, is generated by sympathetic discharges that originate in specific areas of the brainstem. Some groups of neurons located in this region of the central nervous system (CNS), called sympathetic premotor neurons, send direct projections to the sympathetic preganglionic neurons of the intermediolateral (IML) spinal column. The medullary centers responsible for the maintenance and control of cardiovascular tone generally work through reflex cardiovascular mechanisms such as baroreflex, chemoreflex, and cardiopulmonary reflex (3). The terminations of the afferent fibers of the cardiovascular reflexes are located in the nucleus tractus solitarii (NTS) (3). Neurons of the caudal ventrolateral medulla (CVLM) and rostral ventrolateral medulla (RVLM) determine the sympathetic tone, thus contributing to the maintenance of BP and heart rate $(\mathrm{HR})$ at baseline $(4,5)$ and mediate changes in the sympathetic component of the cardiovascular reflexes (6).

Correspondence: M.C. Martins-Pinge, Departamento de Ciências Fisiológicas, Centro de Ciências Biológicas,

Universidade Estadual de Londrina, 86051-990 Londrina, PR, Brasil. Fax: +55-11-3371-4467. E-mail: martinspinge@uel.br

Presented at the XV Simpósio Brasileiro de Fisiologia Cardiovascular, São Paulo, SP, Brazil, February 2-5, 2011.

Received March 2, 2011. Accepted July 28, 2011. Available online August 19, 2011. Published September 16, 2011. 
In two other medullary areas, the nucleus ambiguous (NA) and the dorsal motor nucleus of vagus (DMV), are located the preganglionic parasympathetic neurons (7), which mediate the parasympathetic component of the cardiac reflexes (8).

In addition to the brainstem centers, supramedullary structures are also involved in the control of the cardiovascular system, such as the parabrachial nucleus, Kolliker-Fuse nucleus, the cluster of A5 cells, and the paraventricular nucleus of the hypothalamus (PVN). The latter has been studied among others as one of the main central areas involved in regulating sympathetic outflow because it projects to the IML of the spinal cord where sympathetic premotor neurons are located (9).

One of the situations in which the involvement of the ANS is essential is exercise activity, which promotes cardiovascular regulation, depending on a fine activation of some regions while others are deactivated to guarantee organ perfusion during the execution of exercise. During exercise, there is the need for an increase in cardiac output that involves, besides the intrinsic properties of the heart like the Frank Starling mechanism, also an increase in sympathetic activity to the heart and to arteries, leading to increased levels of heart rate, contractility and vasoconstriction. At the same time, as the blood flow to the active muscles is increased, it tends to decrease in the other tissues. At this level of exercise (aerobic exercise), the mean arterial pressure (MAP) increases a little or may not increase. The exact effects of ANS activity during exercise will depend on the kind of exercise, in addition to other factors.

\section{Cardiovascular and autonomic adaptations to exercise training}

It has been generally accepted that regular physical activity is associated with beneficial effects on the cardiovascular system. Exercise training promotes several cardiovascular adjustments, including remodeling of the heart and skeletal muscle circulation $(10,11)$, improvement of the autonomic control of the heart, and the appearance of resting bradycardia, a characteristic marker of exercise training $(12,13)$. These modulatory effects of training also involve peripheral and central mechanisms.

Trained individuals also exhibit increased maximal oxygen uptake, which is accompanied by several cardiovascular adaptations such as rapid ventricular filling and increased myocardium contractility with larger stroke volume, increased capillary supply, and predominance of arteriole vasodilator responses with larger exercise-induced blood flow. All of these effects favor high oxygen extraction by the exercising muscles. These cardiac and skeletal muscle adaptations help maintain cardiac output with less energy expenditure and thus with a lower heart rate (14).

Regular exercise is also able to modify the cardiac autonomic balance by increasing the parasympathetic activity and decreasing the sympathetic activity (15). Exercise training promotes an increase in the capacity of the ANS to meet the demands of the cardiovascular system, thereby increasing heart rate variability (HRV) (16-18). One of the methods of studying autonomic modulation is by spectral analysis. Spectral analysis is a tool that decomposes cardiovascular signals into their frequency components and quantifies the power of each component (19). Studies of this subject suggest that exercise training causes adaptations in the cardiac autonomic balance, characterized by a predominance of the parasympathetic autonomic component over the sympathetic component. This statement is based on studies that showed that high frequency oscillations are mostly due to autonomic parasympathetic modulation, whereas the low frequency oscillations are derived, at least in part, from the two components of the ANS, i.e., the sympathetic and parasympathetic components (20-22). The genesis of these mechanisms of autonomic cardiovascular control is central, but there is a discussion about the modulation of these mechanisms for interactions of the afferents from the peripheral reflex arc, such as the baroreflex $(23,24)$.

It has been proposed that areas of the CNS, which is known for its involvement in cardiovascular control, appear to show altered activity in response to an exercise session in trained rats (25). Among the areas involved in exercise, the most studied in cardiovascular adaptations are the RVLM (26-28), NTS (29-31), and PVN (15,31-33).

\section{Modulation by brainstem areas}

The importance of the RVLM in the control of the cardiovascular system has been clearly established (3,5,34-36). Both RVLM and CVLM are considered to be important integrative/regulatory regions of cardiovascular activity during static muscle contraction (37), when the pressor reflex of exercise seems to involve the activation of the RVLM neurons $(37,38)$. Considering that the cardiovascular centers participate in the pressor reflex of exercise, and the ANS is modulated after physical training $(10,11,13,39,40)$, exercise training may possibly modulate the RVLM neurons, the major tonic source of supraspinal sympathoexcitatory outflow.

The presence and participation of glutamate, an excitatory amino acid neurotransmitter, in cardiovascular centers have been extensively documented (41-45). This neurotransmitter seems to mediate and/or regulate functions during cardiovascular reflexes, including baroreflexes or chemoreflexes, and muscle pressor responses $(41,46-49)$. The literature documents the role of glutamate within the RVLM during static muscle contraction $(48,49)$. In rats, static muscle contraction elicited an increase in the extracellular concentration of glutamate in the RVLM, suggesting that RVLM-glutamate is involved in the exercise pressor reflex (38). The microinjection of L-glutamate into the RVLM produces an increase in the arterial pressure of awake or anesthetized animals (50).

Although glutamate and other neurotransmitters in the 
RVLM appear to be involved in the cardiovascular response to acute exercise $(37,51)$, the influence of repeated bouts of exercise (chronic exercise) and the recurring, often cyclic, activation of these exercise-related inputs in the RVLM are likely to be important in terms of neuroplastic changes. In a previous study (26), we investigated the effects of a protocol of chronic exercise on the glutamatergic neurotransmission in the RVLM of conscious rats. At this level of physical training, with baseline bradycardia, the glutamate pressor responses in the RVLM produced attenuated increases in MAP compared to control. This blunted effect of L-glutamate microinjection in the RVLM is consistent with an adaptive response to cardiovascular alterations promoted by swimming training, and the mechanism may involve a down-regulation of the glutamate receptors due to chronic exposure of the RVLM neurons to the neurotransmitter.

A similar effect was observed in a 2007 study by Mueller (28), which showed that, compared with physically active animals, sedentary animals (with no repetitive exerciserelated inputs) exhibit enhanced sympathoexcitatory responses, measured by the lumbar nerve activity due to direct glutamatergic activation of the RVLM neurons. These data suggest that alterations occurring in the RVLM may be responsible for enhanced sympathoexcitation in sedentary animals. This effect also does not seem to be caused by a generalized effect on neuronal excitability since responses to angiotensin II microinjection in the RVLM were not enhanced in sedentary animals compared to physically active animals (27).

Another site of great importance for the autonomic regulation is the NTS, where the baroreceptor afferents form the first synapse in the CNS (52). Baroreceptor afferents are tonically active and stimulated by stretching of the aorta and carotid sinus coinciding with the systolic phase of the cardiac cycle. It signals information and sends projections to the NTS in the brain stem, the first synaptic relay of afferents within the CNS. The NTS second-order neurons further stimulate and excite the parasympathetic preganglionic neurons in the DMV and NA, resulting in increased vagal outflow to the heart and bradycardia, and the $y$-aminobutyric acid (GABA)ergic neurons in the CVLM that project to and inhibit neurons within the RVLM, the main premotor nucleus for cardiovascular sympathetic outflow (3). Thus, the NTS is another possible target for modulation of cardiovascular function by exercise.

A study performed on anesthetized rats demonstrated that generalized activation of the NTS with unilateral microinjections of glutamate produced dose-dependent decreases in MAP, HR, and lumbar sympathetic nerve activity (LSNA) that were unaffected by exercise training. Bilateral inhibition of the NTS with the GABA $_{A}$ agonist muscimol produced increases in MAP and LSNA that were blunted by exercise. In contrast, pressor and sympathoexcitatory responses to bilateral microinjections of the ionotropic glutamate receptor antagonist, kynurenic acid, were similar for all groups.
Bradycardic responses to bilateral microinjections of the $\mathrm{GABA}_{A}$ antagonist bicuculline were attenuated by exercise training. These data indicate that alterations in neurotransmission at the level of the NTS significantly contribute to cardiovascular and autonomic regulation after chronic exercise. The authors also suggested that, in addition to alterations in the NTS, the experiments indirectly suggest that changes in other cardiovascular nuclei contribute to the alterations in neural control of circulation observed after exercise training (30).

Literature data have indicated the involvement of oxytocin (OT) in the neural control of the circulation during exercise (53). Oxytocinergic projections from pre-autonomic neurons of the PVN to the NTS and other bulbar areas are involved in both reflex control of the heart and adjustments of the HR during dynamic exercise. Within the solitary-vagal complex (NTS + DMV), OT stimulates autonomic neurons, thereby improving vagal outflow to the heart to augment reflex bradycardia (53). In addition, in normotensive trained rats but not sedentary rats, an acute bout of exercise was accompanied by increased OT content in the dorsal brainstem areas (53), which could help restrain exercise tachycardia in trained individuals. These data suggest that exercise training could activate pathways that are not usually important in sedentary individuals, thereby reinforcing the issue of neural modulation by exercise training.

The renin-angiotensin system (RAS) is a widely distributed regulatory system with hormonal, paracrine, and intracrine functions in many tissues. The brain RAS has been implicated in the pathogenesis of several forms of hypertension, and all the components of the RAS (precursor, enzymes, peptides, and receptors) are present in the brain areas involved in cardiovascular control. Although a pressure fall in hypertension has been associated with the blockade of the central/peripheral RAS, it is not known whether training-induced benefits for cardiovascular control are dependent on changes in the expression/activity of central RAS components. Felix and Michelini (54) observed that hypertension and exercise training affected the expression/activity of brain RAS in opposite directions. Increased arterial pressure and HR are accompanied by overactivity of the brain RAS in spontaneous hypertensive rats (SHR), whereas exercise training blunts RAS activity. The study suggested a similarity in the effects of both low-intensity exercise training and pharmaceutical compounds to block the expression/activity of the RAS precursor within the brain, thus reducing pressure levels. The study reinforces the adoption of exercise training as initiation and/or coadjuvant therapy for prevention/management of hypertension to reduce cardiovascular morbidity and mortality.

The intermediate NTS receives inputs particularly from arterial baroreceptors, but some of these afferents reach the commissural nucleus of the solitary tract (commNTS). The commNTS also receives inputs from arterial chemoreceptors and seems to be highly sensitive to carotid chemore- 
ceptor stimulation. A recent study observed that swimming exercise induced changes in commNTS neurons that were demonstrated by a greater enhancement of hindlimb vasodilatation in WKY vs SHR rats in response to the GABAergic inhibition of these neurons (55). This study reinforces the view that the CNS participates in the cardiovascular adaptations to exercise training and proposes a differential vascular regulation mediated by the CNS.

\section{Modulation by supramedullary areas (hypothalamus)}

The PVN is known to be a place of integration for autonomic and neuroendocrine responses $(31,56)$. Morphological and electrophysiological studies have shown that the PVN is reciprocally connected to other areas of the brain that are involved in cardiovascular function (57). These regions include the NTS, RVLM, and through the lateral spinal thoracolumbar cell column, the location of sympathetic preganglionic neurons $(3,57)$. Through these reciprocal interconnections with the autonomic centers in the brain stem and spinal cord as well as projections to the median eminence and posterior pituitary (neurohypophysis), the PVN is capable of generating complex patterns of neuroendocrine and autonomic regulation, thus controlling homeostasis (see Figure 1).

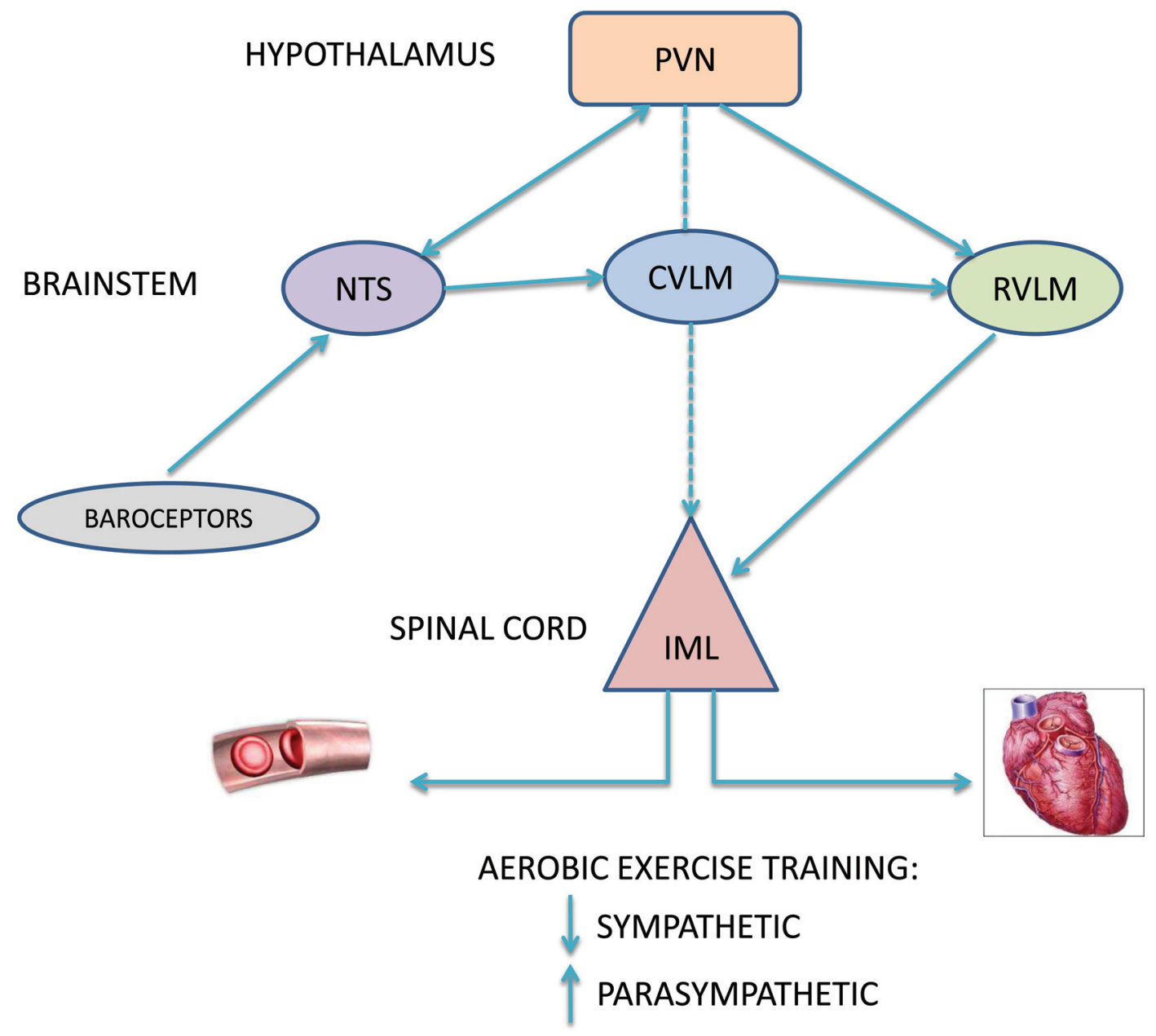

Figure 1. Representation of the hypothalamus and brainstem areas with their projections from baroreceptors, to each other (arrows indicate uni- or bidirectional pathways), and to the intermediolateral column (IML) of the spinal cord. The latter innervates the heart (sympathetic and parasympathetic systems) and blood vessels (only the sympathetic system) to maintain blood pressure and heart rate within normal limits. Aerobic exercise training appears to promote changes in these areas, leading to a lower sympathetic and increased parasympathetic tone. NTS = nucleus tractus solitarii; PVN = paraventricular nucleus of the hypothalamus; CVLM = caudal ventrolateral medulla; RVLM = rostral ventrolateral medulla. 
A characteristic feature of heart failure is increased sympathoexcitation, which correlates with disease severity as well as complications and mortality. The source of the increased sympathoexcitation associated with heart failure is not entirely understood, although several lines of evidence point to a role by the CNS $(57,58)$. In this context, Patel et al. (57) observed that exercise training normalized the potentiated increase in renal sympathetic nerve activity (RSNA) in response to $N$-methyl-D-aspartate (NMDA) microinjected within the PVN of rats with heart failure. The study demonstrated that NR1 receptor expression in the PVN of exercised rats with heart failure was not different from that of sham sedentary or sham-exercised rats. The authors suggested that the normalization of NR1 expression within the PVN is a mechanism by which RSNA responses to NMDA injected into the PVN are normalized by chronic exercise in rats with heart failure. Taken together, these results indicate that a mechanism by which exercise training normalizes sympathetic outflow in heart failure is normalization of glutamatergic mechanisms within the PVN.

A recent study from our laboratory (15) has suggested a contribution of PVN neurons to cardiac autonomic modulation after physical training. After microinjection of bicuculline, a $\mathrm{GABA}_{A}$ antagonist, into the PVN of conscious rats, we did not observe differences in pressor or tachycardic responses between the sedentary and trained groups. However, after the inhibition of PVN neurons with muscimol, a $G_{A B A}$ agonist, the decrease in MAP was enhanced in the trained animals. We also observed that the increased HRV observed at baseline in exercise-trained rats was reversed to control levels when the neurons of the PVN were inhibited by muscimol. Our study suggests that the PVN may be a target for cardiovascular and autonomic modulation by exercise training.

Nitric oxide (NO)-producing neurons are found in the brain areas involved in autonomic activity, including the PVN (59). Moreover, regular physical exercise, which is known to promote a favorable cardiovascular state, augments blood flow and laminar shear stress, resulting in increased NO production and bioavailability (60). In a recent study by our group, we analyzed the involvement of NO in PVN neurons by using the NO-synthase inhibitor, $N$-nitro-L-arginine methyl ester (L-NAME), microinjected into the PVN of sedentary or exercise-trained conscious rats (33). Our data showed that, in sedentary rats, NO inhibition increased arterial pressure and HR to the same extent as in the trained group. However, when we observed the effects on HR and arterial pressure variability, the sedentary group did not show altered autonomic modulation after L-NAME, but the trained group showed increased sympathetic modulation and decreased parasympathetic modulation. The literature has reported that NO-mediated inhibition of RSNA within the PVN was blunted in rats with heart failure, and exercise training improves the altered NO mechanism within the PVN and restores NOmediated changes in the renal sympathetic nerve activity of rats with heart failure (58). On the basis of this evidence, it became clear that the hypothalamus, specially the PVN, may be a good candidate in the CNS for autonomic and cardiovascular modulation by exercise.

In most studies, the perspective of examining the effects of exercise training on cardiovascular variables is based on epidemiological investigations suggesting that physical fitness is beneficial to cardiovascular health. However, it is becoming increasingly evident that a sedentary lifestyle contributes significantly to chronic disease. Therefore, it is important to investigate how normal individuals may benefit by increased exercise activity.

The data of this review suggest that the beneficial effects of physical activity may be due, at least in part, to reductions in sympathetic nervous system activity. Conversely, with the recent association with physical inactivity and chronic disease, these data may also suggest that increased sympathetic nervous system activity contributes to the increased incidence of cardiovascular diseases associated with a sedentary lifestyle.

Sympathetic overactivity is common in many cardiovascular disease states and is related to a higher incidence of morbidity and mortality. Reductions in sympathetic outflow, whether at rest or during conditions that produce sympathoexcitation, may occur following exercise training. Alterations in the cardiovascular regions of the brain stem and other regions that are influenced by the levels of physical activity are likely to play a role in long-term cardiovascular health. Future studies will be important to further identify the central mechanisms involved in physical activity-dependent changes in the control of sympathetic nervous system activity.

\section{References}

1. Irigoyen MC, Moreira ED, Werner A, Ida F, Pires MD, Cestari IA, et al. Aging and baroreflex control of RSNA and heart rate in rats. Am J Physiol Regul Integr Comp Physiol 2000; 279: R1865-R1871.

2. Kenney WL, Munce TA. Invited review: aging and human temperature regulation. J Appl Physiol 2003; 95: 25982603.

3. Dampney RA. Functional organization of central pathways regulating the cardiovascular system. Physiol Rev 1994; 74:
323-364.

4. Guertzenstein PG. Vasodepressor and pressor responses to drugs topically applied to the ventral surface of the brain stem. J Physiol 1972; 224: 84P-85P.

5. Guertzenstein PG, Silver A. Fall in blood pressure produced from discrete regions of the ventral surface of the medulla by glycine and lesions. J Physiol 1974; 242: 489-503.

6. Granata AR, Ruggiero DA, Park DH, Joh TH, Reis DJ. Lesions of epinephrine neurons in the rostral ventrolateral 
medulla abolish the vasodepressor components of baroreflex and cardiopulmonary reflex. Hypertension 1983; 5: V80-V84.

7. McAllen RM, Spyer KM. The location of cardiac vagal preganglionic motoneurones in the medulla of the cat. $J$ Physiol 1976; 258: 187-204.

8. Taylor EW, Jordan D, Coote JH. Central control of the cardiovascular and respiratory systems and their interactions in vertebrates. Physiol Rev 1999; 79: 855-916.

9. Pyner S, Coote JH. Identification of branching paraventricular neurons of the hypothalamus that project to the rostroventrolateral medulla and spinal cord. Neuroscience 2000; 100: 549-556.

10. DiCarlo SE, Bishop VS. Exercise training enhances cardiac afferent inhibition of baroreflex function. Am J Physiol 1990; 258: $\mathrm{H} 212-\mathrm{H} 220$.

11. Mack GW, Thompson CA, Doerr DF, Nadel ER, Convertino VA. Diminished baroreflex control of forearm vascular resistance following training. Med Sci Sports Exerc 1991; 23: 1367-1374.

12. Brum PC, Da Silva GJ, Moreira ED, Ida F, Negrao CE, Krieger EM. Exercise training increases baroreceptor gain sensitivity in normal and hypertensive rats. Hypertension 2000; 36: 1018-1022.

13. Negrao CE, Irigoyen MC, Moreira ED, Brum PC, Freire PM, Krieger EM. Effect of exercise training on RSNA, baroreflex control, and blood pressure responsiveness. Am J Physiol 1993; 265: R365-R370.

14. Clausen JP. Effect of physical training on cardiovascular adjustments to exercise in man. Physiol Rev 1977; 57: 779815.

15. de Abreu SB, Lenhard A, Mehanna A, de Souza HC, Correa FM, Hasser EM, et al. Role of paraventricular nucleus in exercise training-induced autonomic modulation in conscious rats. Auton Neurosci 2009; 148: 28-35.

16. Souza HC, Penteado DM, Martin-Pinge MC, Barbosa NO, Teixeira VP, Blanco JH, et al. Nitric oxide synthesis blockade increases hypertrophy and cardiac fibrosis in rats submitted to aerobic training. Arq Bras Cardiol 2007; 89: 88-104.

17. Souza HC, De Araujo JE, Martins-Pinge MC, Cozza IC, Martins-Dias DP. Nitric oxide synthesis blockade reduced the baroreflex sensitivity in trained rats. Auton Neurosci 2009; 150: 38-44.

18. Tezini GC, Silveira LC, Maida KD, Blanco JH, Souza HC. The effect of ovariectomy on cardiac autonomic control in rats submitted to aerobic physical training. Auton Neurosci 2008; 143: 5-11.

19. Malliani A, Pagani M, Lombardi F, Cerutti S. Cardiovascular neural regulation explored in the frequency domain. Circulation 1991; 84: 482-492.

20. Japundzic N, Grichois ML, Zitoun P, Laude D, Elghozi JL. Spectral analysis of blood pressure and heart rate in conscious rats: effects of autonomic blockers. J Auton Nerv Syst 1990; 30: 91-100.

21. Cerutti C, Gustin MP, Paultre CZ, Lo M, Julien C, Vincent M, et al. Autonomic nervous system and cardiovascular variability in rats: a spectral analysis approach. Am J Physiol 1991; 261: H1292-H1299.

22. Daffonchio A, Franzelli C, Radaelli A, Castiglioni P, Di Rienzo $M$, Mancia $G$, et al. Sympathectomy and cardiovascular spectral components in conscious normotensive rats. Hypertension 1995; 25: 1287-1293.
23. Malliani A. Cardiovascular variability is/is not an index of autonomic control of circulation. J Appl Physiol 2006; 101: 684-688.

24. Eckberg DL. Point:counterpoint: respiratory sinus arrhythmia is due to a central mechanism vs. respiratory sinus arrhythmia is due to the baroreflex mechanism. J Appl Physio/ 2009; 106: $1740-1742$.

25. Ichiyama RM, Gilbert AB, Waldrop TG, Iwamoto GA. Changes in the exercise activation of diencephalic and brainstem cardiorespiratory areas after training. Brain Res 2002; 947: 225-233.

26. Martins-Pinge MC, Becker LK, Garcia MR, Zoccal DB, Neto $\mathrm{RV}$, Basso LS, et al. Attenuated pressor responses to amino acids in the rostral ventrolateral medulla after swimming training in conscious rats. Auton Neurosci 2005; 122: 2128.

27. Becker LK, Santos RA, Campagnole-Santos MJ. Cardiovascular effects of angiotensin II and angiotensin-(1-7) at the RVLM of trained normotensive rats. Brain Res 2005; 1040: 121-128.

28. Mueller PJ. Exercise training attenuates increases in lumbar sympathetic nerve activity produced by stimulation of the rostral ventrolateral medulla. J Appl Physiol 2007; 102: 803813.

29. De Souza CG, Michelini LC, Fior-Chadi DR. Receptor changes in the nucleus tractus solitarii of the rat after exercise training. Med Sci Sports Exerc 2001; 33: 1471-1476.

30. Mueller PJ, Hasser EM. Putative role of the NTS in alterations in neural control of the circulation following exercise training in rats. Am J Physiol Regul Integr Comp Physiol 2006; 290: R383-R392.

31. Michelini LC, Stern JE. Exercise-induced neuronal plasticity in central autonomic networks: role in cardiovascular control. Exp Physiol 2009; 94: 947-960.

32. Jackson K, Silva HM, Zhang W, Michelini LC, Stern JE. Exercise training differentially affects intrinsic excitability of autonomic and neuroendocrine neurons in the hypothalamic paraventricular nucleus. J Neurophysiol 2005; 94: 32113220.

33. Mastelari RB, de Souza HC, Lenhard A, de Aguiar Correa FM, Martins-Pinge MC. Nitric oxide inhibition in paraventricular nucleus on cardiovascular and autonomic modulation after exercise training in unanesthetized rats. Brain Res 2011; 1375: 68-76.

34. McAllen RM, Neil JJ, Loewy AD. Effects of kainic acid applied to the ventral surface of the medulla oblongata on vasomotor tone, the baroreceptor reflex and hypothalamic autonomic responses. Brain Res 1982; 238: 65-76.

35. Cochrane KL, Nathan MA. Pressor systems involved in the maintenance of arterial pressure after lesions of the rostral ventrolateral medulla. J Auton Nerv Syst 1994; 46: 9-18.

36. Reis DJ, Golanov EV, Ruggiero DA, Sun MK. Sympathoexcitatory neurons of the rostral ventrolateral medulla are oxygen sensors and essential elements in the tonic and reflex control of the systemic and cerebral circulations. $J$ Hypertens Suppl 1994; 12: S159-S180.

37. Lillaney R, Maher TJ, Chaiyakul P, Ally A. Changes in extracellular glutamate and pressor response during muscle contraction following AMPA-receptor blockade in the RVLM and CVLM. Brain Res 1999; 844: 164-173.

38. Ally A. Ventrolateral medullary control of cardiovascular activity during muscle contraction. Neurosci Biobehav Rev 
1998; 23: 65-86.

39. Raven PB, Pawelczyk JA. Chronic endurance exercise training: a condition of inadequate blood pressure regulation and reduced tolerance to LBNP. Med Sci Sports Exerc 1993; 25: 713-721.

40. Chen CY, DiCarlo SE. Daily exercise and gender influence arterial baroreflex regulation of heart rate and nerve activity. Am J Physiol 1996; 271: H1840-H1848.

41. Talman WT, Perrone MH, Reis DJ. Evidence for L-glutamate as the neurotransmitter of baroreceptor afferent nerve fibers. Science 1980; 209: 813-815.

42. Benarroch EE, Granata AR, Ruggiero DA, Park DH, Reis DJ. Neurons of $\mathrm{C} 1$ area mediate cardiovascular responses initiated from ventral medullary surface. Am J Physiol 1986; 250: R932-R945.

43. Le Galloudec E, Merahi N, Laguzzi R. Cardiovascular changes induced by the local application of glutamaterelated drugs in the rat nucleus tractus solitarii. Brain Res 1989; 503: 322-325.

44. Bazil MK, Gordon FJ. Spinal NMDA receptors mediate pressor responses evoked from the rostral ventrolateral medulla. Am J Physiol 1991; 260: H267-H275.

45. Kiely JM, Gordon FJ. Role of rostral ventrolateral medulla in centrally mediated pressor responses. Am J Physiol 1994; 267: H1549-H1556.

46. Mitchell JH, Kaufman MP, Iwamoto GA. The exercise pressor reflex: its cardiovascular effects, afferent mechanisms, and central pathways. Annu Rev Physiol 1983; 45: 229242.

47. Kubo T, Amano M, Asari T. N-methyl-D-aspartate receptors but not non-N-methyl-D-aspartate receptors mediate hypertension induced by carotid body chemoreceptor stimulation in the rostral ventrolateral medulla of the rat. Neurosci Lett 1993; 164: 113-116.

48. Caringi D, Maher TJ, Chaiyakul P, Asmundsson G, Ishide T, Ally A. Extracellular glutamate increases in rostral ventrolateral medulla during static muscle contraction. Pflugers Arch 1998; 435: 465-471.

49. Ishide T, Mancini M, Maher TJ, Chayaikul P, Ally A. Rostral ventrolateral medulla opioid receptor activation modulates glutamate release and attenuates the exercise pressor reflex. Brain Res 2000; 865: 177-185.
50. Bachelard H, Gardiner SM, Bennett T. Cardiovascular responses elicited by chemical stimulation of the rostral ventrolateral medulla in conscious, unrestrained rats. J Auton Nerv Syst 1990; 31: 185-190.

51. Reidman DA, Maher TJ, Chaiyakul P, Ally A. Modulation of extracellular glutamate and pressor response to muscle contraction during NMDA-receptor blockade in the rostral ventrolateral medulla. Neurosci Res 2000; 36: 147-156.

52. Irigoyen MC, Krieger EM. Baroreflex control of sympathetic activity in experimental hypertension. Braz J Med Biol Res 1998; 31: 1213-1220.

53. Michelini LC. Oxytocin in the NTS. A new modulator of cardiovascular control during exercise. Ann N Y Acad Sci 2001; 940: 206-220.

54. Felix JV, Michelini LC. Training-induced pressure fall in spontaneously hypertensive rats is associated with reduced angiotensinogen mRNA expression within the nucleus tractus solitarii. Hypertension 2007; 50: 780-785.

55. Ogihara CA, Schoorlemmer GH, Levada AC, Pithon-Curi TC, Curi R, Lopes OU, et al. Exercise changes regional vascular control by commissural NTS in spontaneously hypertensive rats. Am J Physiol Regul Integr Comp Physiol 2010; 299: R291-R297.

56. Coote $\mathrm{JH}$. Cardiovascular function of the paraventricular nucleus of the hypothalamus. Biol Signals 1995; 4: 142149.

57. Kleiber AC, Zheng H, Schultz HD, Peuler JD, Patel KP. Exercise training normalizes enhanced glutamate-mediated sympathetic activation from the PVN in heart failure. Am J Physiol Regul Integr Comp Physiol 2008; 294: R1863R1872.

58. Zheng H, Li YF, Cornish KG, Zucker IH, Patel KP. Exercise training improves endogenous nitric oxide mechanisms within the paraventricular nucleus in rats with heart failure. Am J Physiol Heart Circ Physiol 2005; 288: H2332-H2341.

59. Miyagawa $A$, Okamura $H$, Ibata $Y$. Coexistence of oxytocin and NADPH-diaphorase in magnocellular neurons of the paraventricular and the supraoptic nuclei of the rat hypothalamus. Neurosci Lett 1994; 171: 13-16.

60. Di Francescomarino S, Sciartilli A, Di Valerio V, Di Baldassarre A, Gallina S. The effect of physical exercise on endothelial function. Sports Med 2009; 39: 797-812. 